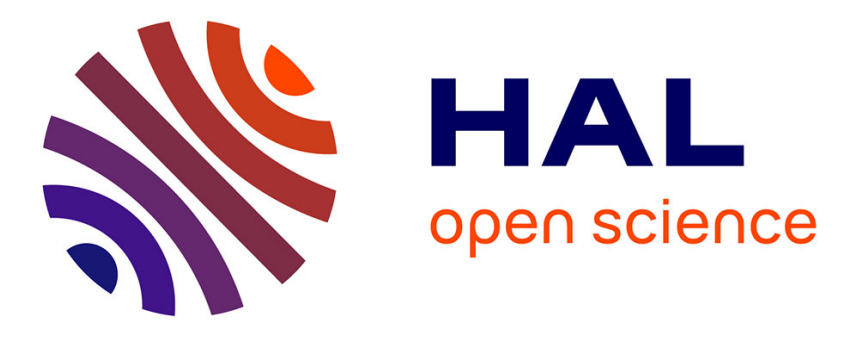

\title{
Super-diffusion in sheared suspensions
}

Mathieu Souzy, Xiaolong Yin, Emmanuel Villermaux, Chérifa Abid, Bloen

Metzger

\section{To cite this version:}

Mathieu Souzy, Xiaolong Yin, Emmanuel Villermaux, Chérifa Abid, Bloen Metzger. Super-diffusion in sheared suspensions. Physics of Fluids, 2015, 27 (4), pp.41705 10.1063/1.4918613 . hal-01449403

\section{HAL Id: hal-01449403 \\ https://hal.science/hal-01449403}

Submitted on 30 Jan 2017

HAL is a multi-disciplinary open access archive for the deposit and dissemination of scientific research documents, whether they are published or not. The documents may come from teaching and research institutions in France or abroad, or from public or private research centers.
L'archive ouverte pluridisciplinaire HAL, est destinée au dépôt et à la diffusion de documents scientifiques de niveau recherche, publiés ou non, émanant des établissements d'enseignement et de recherche français ou étrangers, des laboratoires publics ou privés. 


\section{AIP $\left.\right|_{\substack{\text { Physics of } \\ \text { Fuids }}}$}

\section{Super-diffusion in sheared suspensions}

Mathieu Souzy, Xiaolong Yin, Emmanuel Villermaux, Chérifa Abid, and Bloen Metzger

Citation: Physics of Fluids (1994-present) 27, 041705 (2015); doi: 10.1063/1.4918613

View online: http://dx.doi.org/10.1063/1.4918613

View Table of Contents: http://scitation.aip.org/content/aip/journal/pof2/27/4?ver=pdfcov

Published by the AIP Publishing

\section{Articles you may be interested in}

Effect of shear-induced diffusion on the transfer of heat across a sheared suspension

AIP Conf. Proc. 1542, 1071 (2013); 10.1063/1.4812120

Shear-rate dependent effective thermal conductivity of $\mathrm{H} 2 \mathrm{O}+\mathrm{SiO} 2$ nanofluids

Phys. Fluids 25, 052002 (2013); 10.1063/1.4802049

Roles of particle-wall and particle-particle interactions in highly confined suspensions of spherical particles being sheared at low Reynolds numbers

Phys. Fluids 23, 083302 (2011); 10.1063/1.3613972

Oscillatory shear of suspensions of noncolloidal particles

J. Rheol. 50, 711 (2006); 10.1122/1.2234366

Concentration band dynamics in free-surface Couette flow of a suspension

Phys. Fluids 14, 1580 (2002); 10.1063/1.1460877

\section{Did your publisher get}

18 MILLION DOWNLOADS in 2014?

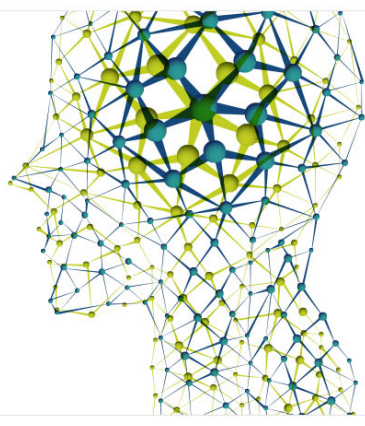




\title{
Super-diffusion in sheared suspensions
}

\author{
Mathieu Souzy, ${ }^{1}$ Xiaolong Yin, ${ }^{2}$ Emmanuel Villermaux, ${ }^{3}$ Chérifa Abid, ${ }^{1}$ \\ and Bloen Metzger ${ }^{1}$ \\ ${ }^{1}$ Aix-Marseille Université, IUSTI-CNRS UMR 7343, 13453 Marseille Cedex 13, France \\ ${ }^{2}$ Petroleum Engineering, Colorado School of Mines, 80401 Colorado, USA \\ ${ }^{3}$ Aix-Marseille Université, IRPHE-CNRS 7342, 13384 Marseille Cedex 13, France
}

(Received 18 December 2014; accepted 3 April 2015; published online 15 April 2015)

\begin{abstract}
We investigate the dispersion of a layer of dye initially applied at the outer wall of a cylindrical Couette-cell into a sheared suspension of non-Brownian spherical particles. The process is directly visualized and quantified at the particle scale. A "rolling-coating" mechanism is found to convectively transport the dye at a constant rate directly from the wall towards the bulk. The fluid velocity fluctuations, $u^{\prime}$, measured with particle image velocimetry, and the imposed shear-rate, $\dot{\gamma}$, are used to define a diffusion coefficient, $D \propto\left\langle u^{\prime} u^{\prime}\right\rangle / \dot{\gamma}$, which is found to increase linearly with the distance from the wall. A solution of the transport equation accounting for this inhomogeneous stirring field describes quantitatively the concentration profiles measured experimentally. It exhibits a super-diffusive character, a consequence of the increase of the stirring strength with distance from the wall. Movies are available with the online version of the paper. (C) 2015 AIP Publishing LLC. [http://dx.doi.org/10.1063/1.4918613]
\end{abstract}

Advection-diffusion coupling and the wide range of length-scales present in turbulent flows lead to the rapid mixing of a scalar field. Conversely, at low Reynolds number, the flow is laminar and mixing, which is then driven solely by molecular diffusion, is extremely slow. This is problematic in numerous situations; practical examples include glass manufacturing, molten polymers, heavy crude oils, industrial processing of food and domestic cooking, pharmaceutical and food industries, and granular materials in civil engineering, to mention a few. How then, can one mix such fluids?

Given the molecular diffusion is extremely slow, mixing enhancement requires conferring to the flow as much complexity and disorder as possible. In the absence of spontaneous inertial instabilities within the fluid such as those occurring in turbulent flows, the only way to complexify the flow is through the boundaries. An archetypal example is having the fluid flow through a porous material: the fixed but tortuous boundaries disrupt the fluid flow which results in a mixing enhancement. ${ }^{1,2}$ Oscillating the fluid flow is also known to enhance mixing. ${ }^{3,4}$ This effect was found to be particularly important in porous materials such as bones. Biologists have shown that through physical activity, periodic load-induced fluid flow in bones is critical for nutrient transport and bone metabolism.,

Another approach consists in moving the boundaries in sequential ways or to carve them in order to force the path lines of the fluid to be chaotic, e.g., in the blinking vortices configuration ${ }^{7}$ or in chaotic mixers for micro-channels. ${ }^{8,9}$

In the present study, we investigate how shearing suspensions of particles enhance the dispersion of a scalar field, prelude to its mixing. The latter is faster when the former is made efficient. Particles, i.e., solid inclusions, behave as moving boundaries. Their permanent re-organisation within the fluid spontaneously promotes mixing even under low Reynolds number conditions. Under flow, particles experience frequent collisions with one another and are thus deviated from their laminar streamlines. This phenomenon called "shear-induced dispersion" has been widely studied in recent years. ${ }^{10-14}$ The particle diffusive motion $D_{p} \propto \dot{\gamma} d^{2}$ where $\dot{\gamma}$ and $d$ denote the imposed shear rate and the particle diameter, respectively. ${ }^{15}$ This scaling is expected since the only time scale in the system is the inverse of the shear rate and the relevant length scale, i.e., the characteristic length scale of the fluid velocity disturbances is given by the particle size. Particles in essence can be thought of as many 
"stirrers": they confer to the flow a stochastic component of advection that produces an efficient mixing. Recent experimental results ${ }^{16}$ demonstrated that the transfer of heat across a suspension of particles is substantially ( $>200 \%$ ) increased when the suspension is sheared. Other studies ${ }^{17,18}$ investigated the transfer of mass across a sheared suspension of particles using an electrochemical method to measure the macroscopic rate of augmented transport of solutes. These studies are very promising in terms of applications. However, from a fundamental point of view, many questions remain open. Specifically, there are to our knowledge, no measurements of the mixing process performed at the particle scale. Yet, this information is crucial to understand the mechanisms at the origin of the transfer intensification. This point constitutes the motivation of the present study. Following this aim, we developed an original experimental setup that allows direct visualization of the dispersion process of a layer of dye in a sheared suspension of non-Brownian particles.

The experimental setup is sketched in Figure 1. The inner cylinder that has a radius of $5 \mathrm{~cm}$ and a height of $12 \mathrm{~cm}$ is driven by a precision rotating stage (M-0.61.PD from PI piezo-nano positioning) with high angular resolution $\left(3 \times 10^{-5} \mathrm{rad}\right)$. The transparent PMMA outer cylinder is stationary and the gap between the two cylinders is $L_{g a p}=12 \mathrm{~mm}$. The temperature of the whole setup is controllable $\left( \pm 0.05^{\circ} \mathrm{C}\right)$ as the Couette cell is embedded in a square water-bath jacket connected to a cryo-thermostat. More details about the setup are provided in Metzger et al. ${ }^{16}$

a)

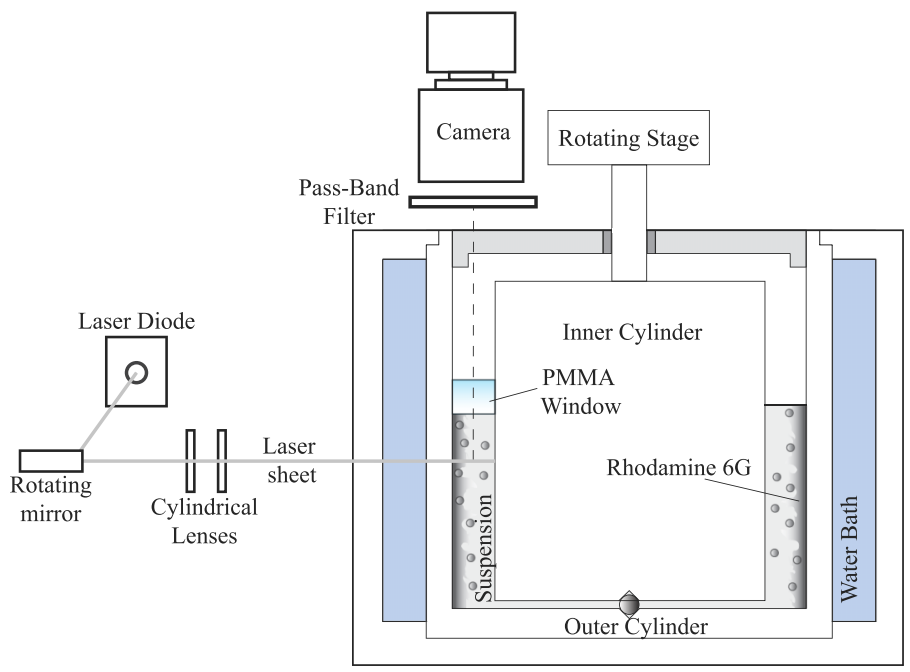

b)
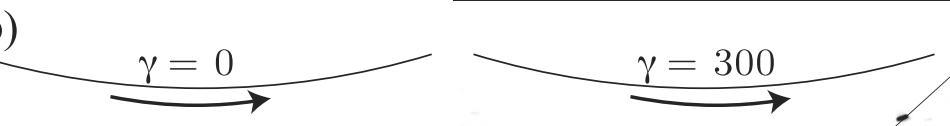

c)
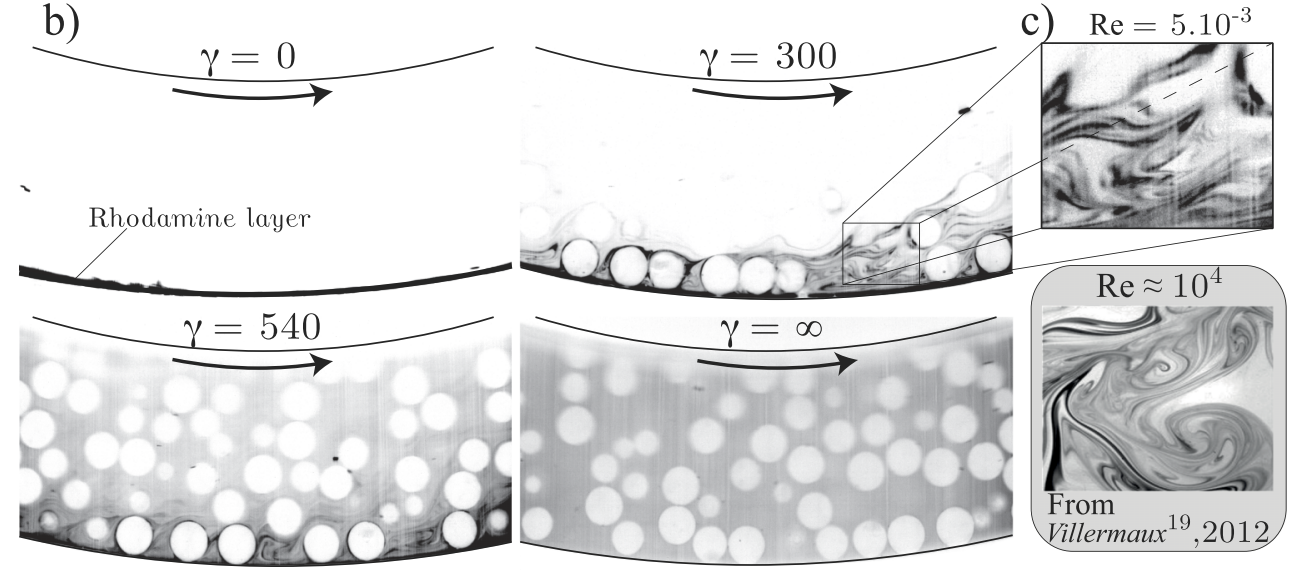

FIG. 1. (a) Sketch of the experimental setup. (b) Successive snapshots of the suspension. Initially, a thin layer of Rhodamine 6G (in black) is located on the outer cylinder. When the suspension is sheared, the dye progressively spreads across the cell-gap until homogeneity is reached. Particles appear as white discs. See Movie 1 in the supplementary material ${ }^{24}$ for detailed evolution of the layer of dye. (c) Close-up view of the flow pattern. Note that this mixing pattern is reminiscent to those observed in turbulent flows, ${ }^{20}$ see bottom picture. 
The fluid is a Newtonian mixture of Triton X-100, zinc chloride, and water. Its viscosity is $\eta=3 \mathrm{~Pa}$ s so that inertial effects can be neglected (the Reynolds number $R e=5 \times 10^{-3}$ ). The composition of the fluid is also chosen to match both the density of the particles $\left(\rho_{f}=\rho_{\text {part }}=1.19 \mathrm{~g} \mathrm{~cm}^{-3}\right)$ to avoid sedimentation and their index of refraction to obtain an optically transparent suspension. ${ }^{19}$ A small amount of hydrochloric acid $(\approx 0.05 \mathrm{wt} . \%)$ is added to the solution to prevent the formation of zinc hypochlorite precipitate, significantly improving the transparency of the mixture. Finally, the temperature of the water-bath is adjusted in order to finely tune index-matching between the fluid and the particles (best index-matching was obtained for $T \approx 18.5^{\circ} \mathrm{C}$ ). We use spherical PMMA beads from Engineering Laboratories, Inc., with a diameter $d=2 \mathrm{~mm}$. These particles were specially chosen for their high surface quality and good transparency. All experiments are performed at a volume fraction $\phi=35 \%$.

As presented in Figure 1, the rhodamine fluoresces under illumination provided by a thin horizontal laser sheet. Images are acquired from the top using a high resolution camera (Basler Scout) and a high-pass filter $(\lambda>550 \mathrm{~nm})$ is used to eliminate direct light reflexions due to the imperfect index-matching. The protocol is the following: a thin layer of fluid doped with Rhodamine $6 \mathrm{G}$ $\left(C_{\text {rhod }}=9 \times 10^{-7} \mathrm{~g} \mathrm{ml}^{-1}\right)$ is homogeneously applied on the outer cylinder using a brush. Then, the gap is carefully loaded with the suspension without disturbing the rhodamine layer applied on the outer cylinder. After waiting $30 \mathrm{~min}$ to reach thermal equilibrium (thus optimal index-matching), the suspension is sheared at a fixed shear-rate, $\dot{\gamma}=0.5 \mathrm{~s}^{-1}$. The evolution of the mean dye concentration profiles across the gap is obtained by averaging the light intensity re-emitted by the Rhodamine $6 \mathrm{G}$ along the flow direction. In total, four experiments were performed for which the whole protocol was repeated entirely.

We performed Particle Image Velocimetry (PIV) to measure the velocity fluctuations of the fluid phase. The fluid was seeded with passive fluorescent tracers (MF-Rhodamine B-particles 3, $23 \mu \mathrm{m}$ ). Images were acquired into burst of five images separated by a strain interval $\gamma_{0}=0.02$. A total of 200 bursts, separated by a strain interval $\gamma \approx 10$, were acquired in order to obtain averages over a large ensemble of statistically uncorrelated configurations. Note that we do not report the velocity fluctuations near the inner (mobile) since it is very difficult to measure velocity fluctuations in regions of the flow where the mean velocity is large.

When shearing the suspension, the rhodamine layer initially applied on the outer cylinder progressively spreads into the gap until homogeneity is reached, as seen in Figure 1(b). Two qualitative yet important observations can be made. First, if no particles had been introduced into the fluid, thus having a dispersion process solely driven by molecular diffusion $\left(D_{0} \approx 10^{-13} \mathrm{~m}^{2} \mathrm{~s}^{-1}\right.$, see Culbertson et $\left.a l^{21}\right)$, the time scale to reach homogeneity, $t=L_{\text {gap }}^{2} / D_{0}$, would have been of the order of $30 \mathrm{yr}$. Here, in the sheared suspension, homogeneity is reached after $30 \mathrm{~min}$. The transfer enhancement is thus extremely large. We show in the sequel that the reason is that the dye dispersion process is super-diffusive but, if from a naive comparison of the timescales above one would define an effective coefficient, it would be such that $D_{\text {eff }} / D_{0} \approx 10000$. Second, the patterns observed in the mixing layer, shown on Figure 1(c), strikingly remind those observed in turbulent mixing. For comparison, a picture from earlier work ${ }^{20}$ of a blob of dye stirred in a turbulent flow at a Reynolds number $R e \approx 10^{4}$ is shown on Figure 1(c). In the suspension, even though the Reynolds number is small $\left(\operatorname{Re}=5 \times 10^{-3}\right)$, the fluid velocity disturbances generated by the particles contribute to accelerate the dispersion process.

The dye concentration profiles as a function of the distance from the wall measured at successive strains, $\gamma=\dot{\gamma} t$, are shown on Figure 2(a). Their overall shape is interestingly analogous to the temperature profiles measured in turbulent Rayleigh-Benard convection. ${ }^{22}$ In the latter configuration, the temperature is very homogeneous in the bulk thanks to the efficient mixing induced by the numerous thermal eddies. Conversely, strong temperature gradients are observed near the walls, in the thermal boundary layers, where heat transfer only occurs through thermal diffusion. Our system shows similar trends. Concentration profiles are flat in the bulk, suggesting that an efficient dispersion mechanism is at play in that region and a strong concentration gradient is observed close to the wall where the transfer of dye appears to be much slower. This is consistent with the scenario proposed by Wang et al. ${ }^{18}$ that the particle shear-induced diffusivity drives the mixing process: in the bulk, after each collision, particles are free to translate in any direction. Conversely, particles located against the wall are sterically frustrated: when approaching the walls, their translational diffusivity must vanish. For comparison, the 

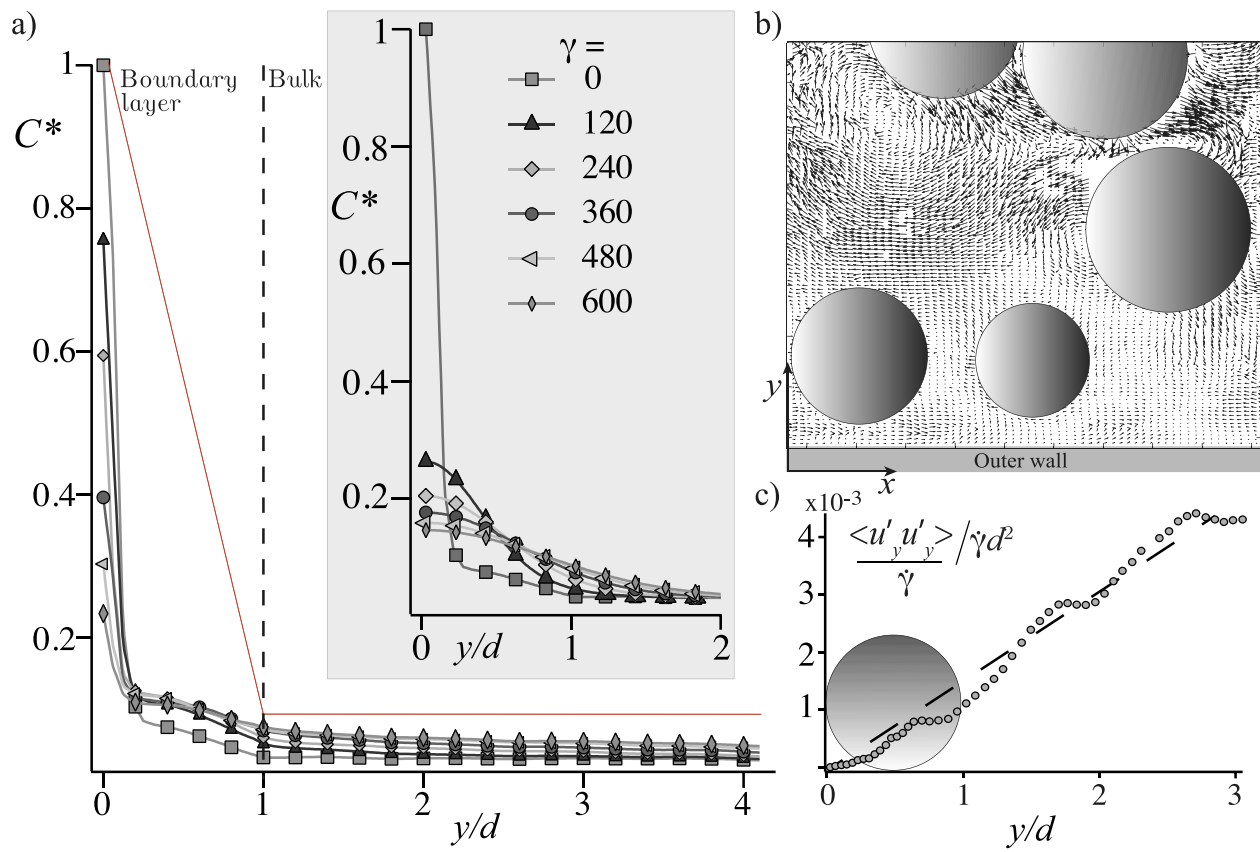

FIG. 2. (a) Concentration profiles versus distance from the wall, $y / d$ at successive strains $\gamma=\dot{\gamma} t$. Inset: Concentration profiles one would obtain for a homogenous diffusion coefficient equal to $D_{\text {eff. }}$ (b) Example of velocity-fluctuations field (the affine part of the flow was subtracted). See Movie 2 in the supplementary material ${ }^{24}$ for raw images of the flowing suspension. (c) Dimensionless diffusion profile, $\frac{\left\langle u_{y}^{\prime} u_{y}^{\prime}\right\rangle}{\dot{\gamma}} / \dot{\gamma} d^{2}$, versus distance from the wall, $y / d$.

inset of Figure 2(a) shows the concentration profiles one would obtain for a homogeneous diffusion coefficient equal to $D_{\text {eff }}$. In that case, the concentration at the wall decays much faster.

To investigate how the dye is transported from the wall towards the bulk, we performed PIV measurement of the fluid velocity field. Owing to the extremely small value of the molecular diffusion coefficient $D_{0}$, this approach seems reasonable since the process we are investigating is likely to be a dispersion problem dominated by advection, i.e., the stirring motions caused by the particles themselves. A typical velocity fluctuation field is illustrated in Figure 2(b). Dimensionally, these velocity fluctuations can be used to build a diffusion coefficient, $D(y) \propto\left\langle u_{y}^{\prime} u_{y}^{\prime}\right\rangle / \dot{\gamma}$, quantifying the magnitude of the velocity disturbances induced by the particles. While existing models ${ }^{23}$ proposed that the diffusion coefficient should increase as $y^{4}$, our experimental observations suggest a linear increase with the distance from the wall, namely, $D(y)=U y$, with $U$ a constant velocity (Figure 2(c)). Note that for $y / d<1$, a significant amount of fluctuations is present in the fluid, although in that region, as we mentioned before, the particle translational diffusivity must be zero. As can be seen in Movie 3 in the supplementary material, ${ }^{24}$ particles located against the wall rotate within the shear flow; this rotation induces disturbances within the fluid.

This close examination of the boundary layer also reveals an unexpected transport-enhancing mechanism: as shown on Figure 3(a), by rolling against the wall, particles get coated with a thin layer of dye. The particle rotation thus contributes to a convective transport of the rhodamine located on the wall directly towards the bulk. This mechanism is crucial since it breaks the initially expected diffusive boundary layer. ${ }^{18}$ Furthermore, when computing the total amount of rhodamine present in the bulk, $m=\int_{d}^{3 d} C(y, t) \mathrm{d} y$, we find this quantity increases linearly with time or equivalently with strain, $m \propto t$, see Figure 3(b). This can easily be explained assuming the initial layer of rhodamine applied on the outer wall constitutes an infinite reservoir of dye. Since the particle rotation rate is fixed by the shear rate and calling $\delta$ the thickness of the rhodamine layer coating the particles, we expect this "rolling-coating" mechanism to produce a constant mass flux, $J=\delta \dot{\gamma}$, of rhodamine towards the bulk. This scenario indeed leads to a linear growth of the total amount of rhodamine present in the bulk, $m=\int_{0}^{t} J d t^{\prime}=\delta \dot{\gamma} t$. Note that being driven by the rotation of the particles in the layer contacting the wall, the mass flux is de facto identical at $y=0$ and $y=d$. 

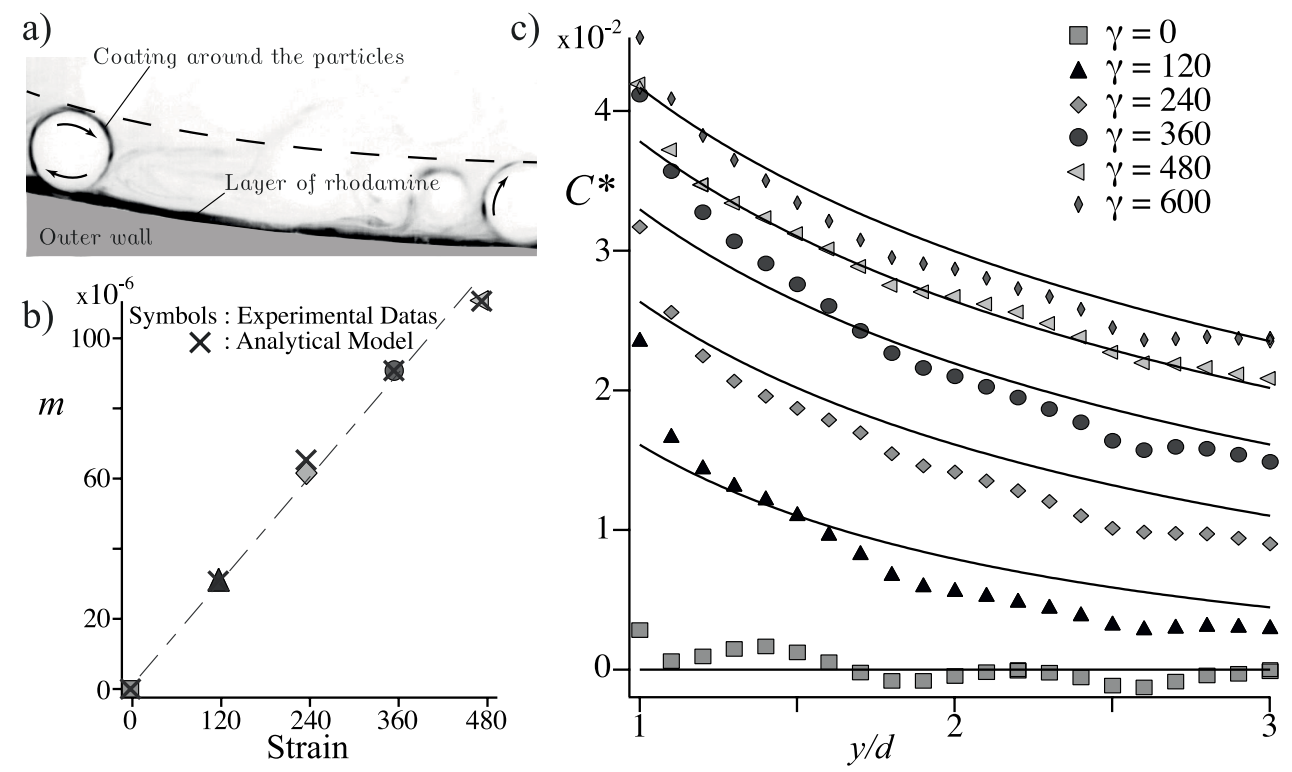

FIG. 3. (a) Close view of the "rolling-coating" mechanism. See also Movie 3 in the supplementary material. ${ }^{24}$ (b) $m=$ $\int_{d}^{3 d} C(y, \gamma) d y$ for the experimental profile and the analytical solution, for successive strains. (c) Comparison between the experimentally measured concentration profiles (markers) and the analytical solution (full line, see Eq. (2)) versus distance from the wall, $y / d$.

Thus, considering the linear increase of the diffusion coefficient with the distance from the wall, the transport equation for the dye mean concentration field $C(y, t)$ becomes

$$
\begin{aligned}
& \frac{\partial C}{\partial t}=\frac{\partial}{\partial y}\left(D(y) \frac{\partial C}{\partial y}\right), \\
& D(y)=U y,
\end{aligned}
$$

and can be solved assuming a constant mass flux as a boundary condition at $y=0$. The solution is given by an incomplete gamma function

$$
C(y, t)=\frac{J}{U} \Gamma\left(0, \frac{y}{U t}\right)=\frac{J}{U} \int_{\frac{y}{U t}}^{\infty} \frac{e^{-x}}{x} \mathrm{~d} x .
$$

This solution closely matches the experimental concentration profiles recorded in the bulk, when the parameter $J$ is adjusted such that $\int_{d}^{3 d} \frac{J}{U} \Gamma\left(0, \frac{y}{U t}\right) \mathrm{d} y=m$, ensuring that the amount of dye in the range $[d-3 d]$ coincides with the experimental concentration profiles, see Figure $3(b)$. Note that the integral of the model should not grow linearly over that restricted range (only the total mass should), yet a linear assumption is reasonable. This solution also demonstrates, not surprisingly considering the non-constancy of $D(y)$ with $y$, that the transport is enhanced, the dye being removed from the wall all the more rapidly that it is distant from it. Indeed, the penetration distance (typical width of the concentration profile) of the dye is $\int y C(y, t) \mathrm{d} y \sim m U t=J U t^{2}$, a clearly super-diffusive dispersion law. This shows the strong effect of the presence of the wall, and of the particles, on the overall transfer process. The parameter $U$ is found to be equal to $2.12 \times 10^{-6} \mathrm{~m} \mathrm{~s}^{-1}$ which leads to $D(y) / \dot{\gamma} d^{2}=$ $2.12 \times 10^{-3} \mathrm{y} / \mathrm{d}$. At distances from the wall around 3 to 4 beads diameters, the diffusion coefficient $D(3 d) \approx 10^{-2} \dot{\gamma} d^{2}$ which matches earlier bulk measurements ${ }^{10,16}$ of the particle diffusion coefficient at this concentration. This also leads to $D(y)=1.30\left\langle u_{y}^{\prime} u_{y}^{\prime}\right\rangle / \dot{\gamma}$ suggesting the dimensional approach based on the fluid velocity fluctuations provides a correct estimate of the particles diffusion coefficient.

Sheared suspensions happen to be a system where an efficient dispersion spontaneously occurs even under low Reynolds number conditions. Using planar laser-induced fluorescence, we directly visualized the spread of a layer of rhodamine initially applied on the outer wall of a cylindrical Couette-cell. For the flow conditions studied here $\left(R e=5 \times 10^{-3}\right.$ and $\left.P e=\dot{\gamma} d^{2} / D_{0}=10^{7}\right)$, the dispersion of the dye has been shown to be super-diffusive close to the wall, leading to a transport four 
orders of magnitude more efficient than its molecular expectation in the fluid at rest. The dispersion coefficient in the bulk is of the same order of magnitude as the particle diffusion coefficient measured in earlier works. ${ }^{10,16}$ The shape of the mean concentration profiles reflects an increase of the stirring intensity with distance to the wall, the sheared particles responsible for these motions being sterically damped at the wall. High-resolution PIV measurements of the fluid velocity disturbances generated by the particles have shown that their diffusion coefficient increases approximately linearly with the distance from the wall. The solution of the transport equation with a linear variation of the diffusion coefficient in space and a constant mass flux at the wall boundary condition provided concentration profiles in good agreement with the ones measured experimentally. However, mass transfer at the wall not only occurs through molecular diffusion. Direct visualization of the mixing process allowed us to identify a "rolling-coating" mechanism: particles rotate against the wall and get coated with a thin layer of dye. The particles convect, at a constant rate, the dye from the wall directly towards the bulk (see Movie 3 in the supplementary material ${ }^{24}$ ) breaking the diffusive boundary layer. In future experiments, we plan to vary the particle size and shear-rate to explore a large range of Péclet number and deeper range of distances from the wall.

We would like to thank Paul Cervetti, Sady Noel, and Stephane Martinez for building the experimental setup. This work was supported by ANR JCJC SIMI 9 and by the Labex MEC ANR-11LABX-0092 and A*MIDEX ANR-11-IDEX-0001-02LABEX MEC.

${ }^{1}$ T. Le Borgne, M. Dentz, D. Bolster, J. Carrerra, J.-R. Dreuzy, and P. Davy, "Non-Fickian mixing: Temporal evolution of the scalar dissipation rate in heterogeneous porous media," Adv. Water Resour. 33(12), 1468-1475 (2010).

2 E. Villermaux, "Mixing by porous media," C. R. Mec. 340(11), 933-943 (2012).

${ }^{3}$ G. Taylor, "Dispersion of soluble matter in solvent flowing slowly through a tube," Proc. R. Soc. A 219(1137), 186-203 (1953).

${ }^{4}$ R. Aris, "On the dispersion of a solute in pulsating flow through a tube," Proc. R. Soc. A 259(1298), 370-376 (1960).

${ }^{5}$ C. Price, X. Zhou, W. Li, and L. Wang, "Real-time measurement of solute transport within the lacunar-canalicular system of mechanically loaded bone: Direct evidence for load-induced fluid flow," J. Bone Miner. Res. 26(2), 277-285 (2011).

${ }^{6}$ S. M. Schmidt, M. J. McCready, and A. E. Ostafin, "Effect of oscillating fluid shear on solute transport in cortical bone," J. Biomech. 38(12), 2337-2343 (2005).

${ }^{7}$ A. Daitche and T. Tél, "Dynamics of blinking vortices," Phys. Rev. E 79(1), 016210 (2009).

${ }^{8}$ A. D. Stroock, S. K. W. Dertinger, A. Ajdari, I. Mezic, H. A. Stone, and G. M. Whitesides, "Chaotic mixer for microchannels," Science 295(5555), 647-651 (2002).

${ }^{9}$ E. Villermaux, A. D. Stroock, and H. A. Stone, "Bridging kinematics and concentration content in a chaotic micromixer," Phys. Rev. E 77, 015301R (2008).

${ }^{10}$ A. Sierou and J. F. Brady, "Shear-induced self-diffusion in non-colloidal suspensions," J. Fluid Mech. 506, 285-314 (2004).

${ }^{11}$ V. Breedveld, D. Van den Ende, M. Bosscher, R. J. J. Jongschaap, and J. Mellema, "Measurement of the full shear-induced self-diffusion tensor of noncolloidal suspensions," J. Chem. Phys. 116, 23 (2002).

${ }^{12}$ F. R. Da Cunha and E. J. Hinch, "Shear-induced dispersion in a dilute suspension of rough spheres," J. Fluid Mech. 309, 211-223 (1996).

${ }^{13}$ E. C. Eckstein, D. G. Bailey, and A. H. Shapiro, "Self-diffusion of particles in shear flow of a suspension,” J. Fluid Mech. 79, 191-208 (1977).

${ }^{14}$ P. A. Arp and S. G. Mason, "The kinetics of flowing dispersions IX. Doublets of rigid spheres (Experimental)," J. Colloid Interface Sci. 61(4095), 44-61 (1976).

${ }^{15}$ D. Leighton and A. Acrivos, "Measurement of shear-induced self-diffusion in concentrated suspensions of spheres," J. Fluid Mech. 177, 109-131 (1987).

${ }^{16}$ B. Metzger, O. Rahli, and X. Yin, "Heat transfer across sheared suspensions: Role of the shear-induced diffusion," J. Fluid Mech. 724, 527-552 (2013).

${ }^{17}$ N. H. L. Wang and K. H. Keller, "Augmented transport of extracellular solutes in concentrated erythrocyte suspensions in Couette flow," J. Colloid Interface Sci. 103(1), 210-225 (1985).

${ }^{18}$ L. Wang, D. L. Koch, X. Yin, and C. Cohen, "Hydrodynamic diffusion and mass transfer across a sheared suspension of neutrally buoyant spheres," Phys. Fluids 21(3), 033303 (2009).

19 J. A. Dijksman, F. Rietz, K. A. Lorincz, M. van Hecke, and W. Losert, "Invited article: Refractive index matched scanning of dense granular materials," Rev. Sci. Instrum. 83(1), 011301 (2012).

${ }^{20}$ E. Villermaux, "On dissipation in stirred mixtures," Adv. Appl. Mech. 45, 91-107 (2012).

${ }^{21}$ C. T. Culbertson, S. C. Jacobson, and J. M. Ramsey, "Diffusion coefficient measurements in microfluidic devices," Talanta 56, 365-373 (2002).

${ }^{22}$ F. Chilla, S. Ciliberto, C. Innocenti, and E. Pampaloni, "Boundary layer and scaling properties in turbulent thermal convection," Il Nuovo Cimento 15 D(09), 1229-1249 (1993).

${ }^{23}$ D. L. Koch, "Hydrodynamic diffusion near solid boundaries with applications to heat and mass transport into sheared suspensions and fixed-fibre beds," J. Fluid Mech. 318, 31-47 (1996).

${ }^{24}$ See supplementary material at http://dx.doi.org/10.1063/1.4918613 for three movies: Movie 1) Dispersion of a rhodamine layer in a sheared suspension of particles, Movie 2) PIV in the flowing suspension, and Movie 3) Rolling-coating mechanism. 